\title{
On the Inherent Variability of Particulate Matter Concentrations on Small Scales and the Consequences for Miniaturized Particle Sensors
}

\author{
Paul Maierhofer ${ }^{*}$, Georg Röhrer ${ }^{2}$, Markus Bainschab ${ }^{1}$, Alexander Bergmann' \\ ${ }^{1}$ Institute of Electronic Sensor Systems, Graz University of Technology, 8010 Graz, Austria \\ ${ }^{2}$ ams AG, 8141 Premstätten, Austria
}

\begin{abstract}
Despite all the evident benefits of miniaturized particulate matter (PM) sensors, an inherent drawback exists in the uncertainty and validity of the measurement, which is closely related to the discrete nature of particulates suspended in air. The miniaturization of these devices not only leads to a smaller footprint for the devices themselves but also to a smaller volume of air being sampled. Even if a perfect measurement system is assumed, an uncertainty lies in assigning a supposedly representative particle concentration value to an environment due to the inherent variability of PM concentrations on small scales. This stems from the fact that particles are stochastically distributed in the air, leading to a non-uniform concentration for arbitrarily small volumes. Consequently, an uncertainty exists according to counting statistics, as the number of investigated particles in a small air sample is also low. Depending on the metric, the uncertainty may be augmented, as a small number of particles cannot accurately capture the distribution of particle sizes, especially since the size distribution extends over several orders of magnitude. This distribution related uncertainty is relevant for surface and mass related metrics in addition to the uncertainty resulting from counting statistics. We detected a minor impact from the distribution of the particle mass density, which contributes to the uncertainty for mass-related metrics, such as $\mathrm{PM}_{1}, \mathrm{PM}_{2.5}$ and $\mathrm{PM}_{10}$. We investigated the expected measurement uncertainty by analytical means and concluded that the distribution of particle sizes, the sample size and the ambient particle concentration significantly affect the measurement uncertainty for the range of conditions considered. To the best of our knowledge, this uncertainty has not been discussed in the current literature.
\end{abstract}

Keywords: Aerosol sampling; Measurement uncertainty; Miniaturized sensors.

\section{INTRODUCTION}

Serious adverse health effects of particulate matter (PM) on the human body (Ranft et al., 2009; Kioumourtzoglou et al., 2016) raise a public desire for highly integrated, cost-effective and easy-to-use PM sensors. As the chase for smaller and smaller PM sensors is currently at an alltime high (Paprotny et al., 2013; Li et al., 2014; Ciccarella et al., 2016; Bao et al., 2018; Hapidin et al., 2019), it is not only fruitful but also necessary to have a closer look at limitations we encounter. The miniaturization of microfluidic devices, such as miniaturized PM sensors, yields lower flow rates compared to available macroscopic devices, e.g., Papronty et al. (2013) report a flow rate of only $6 \mathrm{~mL} \mathrm{~min}^{-1}$. Therefore, the sample size of PM sensors is typically decreasing for a given integration time as they get smaller.

\footnotetext{
${ }^{*}$ Corresponding author.

Tel.: 0043-316-873-3350

E-mail address: paul.maierhofer@tugraz.at
}

As the sampled volume gets smaller, also the number of particles analyzed and consequently used to estimate ambient PM concentrations gets lower.

The target of this work is to evaluate the impact of miniaturization on the validity of the measurements, neglecting all possible imperfections of a sensor. The uncertainty of the estimated ambient particle concentration given the measurement of a sensor is equal to the inherent variability of PM on the scale of the sample volume. We find three separate contributions to the total uncertainty: One stems from counting statistics, the second one is caused by the broad distribution of particle sizes in the ambient environment, which plays a role for metrics such as surface concentration and mass concentration, and a third contribution to the total measurement uncertainty comes from the distribution of particle mass densities. A small number of particles does not represent this complex situation ideally. The smaller the sample gets, the higher the variability between measurements within the same ambient conditions will be. This uncertainty is also dependent on the metric used, which can depend on the sensor effect in use.

The discussed uncertainty is not covered by the current 
literature to the best of our knowledge.

\section{METHODS}

We state the analytical expressions of three investigated metrics in terms of the relevant particle properties as shown in Eq. (1). We use these analytical expressions to find the expectation value and the variance of the metrics on small volumes.

We can write the particulate concentrations within a sample volume as PN, PS and $\mathrm{PM}_{\mathrm{x}}$, as in Eq. (1). PN is the particle number concentration. PS corresponds to the total particle surface area per volume. $\mathrm{PM}_{\mathrm{x}}$ is a placeholder for the most used mass metrics, such as $\mathrm{PM}_{1}, \mathrm{PM}_{2.5}$ or $\mathrm{PM}_{10}$. These metrics count the total mass of particles up to a diameter indicated after "PM" in units of $\mu \mathrm{m}$. For example, $\mathrm{PM}_{1}$ corresponds to the total mass of particles less than $1 \mu \mathrm{m}$ in diameter per cubic meter.

$$
\begin{aligned}
& P N=\frac{1}{V_{\text {Sample }}} \sum_{n=1}^{N} \frac{N}{V_{\text {Sample }}} \\
& P S=\frac{1}{V_{\text {Sample }}} \sum_{n=1}^{N} S_{n} \\
& \mathrm{PM}_{\mathrm{x}}=\frac{1}{V_{\text {Sample }}} \sum_{n=1}^{N} V_{n} \rho_{n}
\end{aligned}
$$

where $\mathrm{N}$ is the total number of particles within the sample, and $S_{n}, V_{n}$ and $\rho_{n}$ are the surface, volume and the mass density of the $\mathrm{n}^{\text {th }}$ particle, respectively. $\mathrm{V}_{\text {Sample }}$ denotes the sampled volume of air. Mathematically speaking, $\mathrm{N}$ is a random positive integer number, and $S_{n}, V_{n}$ and $\rho_{n}$ are each independent and identically distributed random variables, which means that, e.g., all $\mathrm{V}_{\mathrm{n}}$ have the same probability distribution, with finite expectation value and variance.

For the particle number concentration, only the number of particles per volume is relevant, whereas for the surface concentration, the individual surfaces of the single particles are summed up and for mass concentrations, their masses. This concentration will, especially for small sample volumes, vary from the mean ambient particle concentration since the suspended particles are discrete objects. In an extreme case, we could think of a situation where only one particle is suspended in a known volume of air. We can assume a sensor, which would need a large number (m) of measurements to analyze the whole air in the total volume. This sensor would report in $\mathrm{m}-1$ measurements a concentration of 0 and in one measurement, a concentration of $\mathrm{m}$ times the mean concentration in said volume. Clearly, this sensor would not be able to capture the situation with only one measurement - not because of imperfections of the sensor itself but because of the high variability in the particle concentration. Another effect stems from the size distribution and the mass density distribution of particles, which complicates the situation for surface concentration and mass concentration metrics.

A sensor will use the measurement value of the sampled volume to estimate the particle concentration on a larger scale. Therefore, we are interested in the expectation value, $<>$, and the variance, $\operatorname{Var}()$, for each of the investigated metrics, which we calculate using Wald's equation as well as Blackwell-Girshick's equation (Klenke, 2013) and the assumption that the number of particles $(\mathrm{N})$, the surface $(\mathrm{S})$, the volume $(\mathrm{V})$ and the density $(\rho)$ are uncorrelated.

$$
\begin{aligned}
& \langle P N\rangle=\langle N\rangle \frac{1}{V_{\text {Sample }}} \\
& \langle P S\rangle=\langle N\rangle\langle S\rangle \frac{1}{V_{\text {Sample }}} \\
& \left\langle\mathrm{PM}_{\mathrm{x}}\right\rangle=\langle N\rangle\langle V\rangle\langle\rho\rangle \frac{1}{V_{\text {Sample }}} \\
& \operatorname{Var}(P N)=\operatorname{Var}(N) \times\left(\frac{1}{V_{\text {Sample }}}\right)^{2} \\
& \operatorname{Var}(\mathrm{PS})=\left(\operatorname{Var}(\mathrm{N})\langle\mathrm{S}\rangle^{2}+\operatorname{Var}(\mathrm{S})\langle\mathrm{N}\rangle\right) \times\left(\frac{1}{\mathrm{~V}_{\text {Sample }}}\right)^{2} \\
& \operatorname{Var}\left(\mathrm{PM}{ }_{\mathrm{x}}\right)=\left(\begin{array}{l}
\langle\mathrm{V}\rangle^{2}\langle\rho\rangle^{2} \operatorname{Var}(\mathrm{N})+\langle\rho\rangle^{2}\langle\mathrm{~N}\rangle \operatorname{Var}(\mathrm{V})+ \\
\langle\mathrm{V}\rangle^{2}\langle\mathrm{~N}\rangle \operatorname{Var}(\rho) \\
\times\left(\frac{1}{\mathrm{~V}_{\text {Sample }}}\right)^{2}
\end{array}\right.
\end{aligned}
$$

Eq. (3) shows the total variance of each metric's value. For PN, the situation is straightforward since only the counting uncertainty plays a role. The surface concentration has an additional term including the variance of a single particle's surface. $\mathrm{PM}_{\mathrm{x}}$ has a term proportional to the variance of a single particle's volume, $\operatorname{Var}(\mathrm{V})$, as well as a term with the variance of the mass density, $\operatorname{Var}(\rho)$. $\operatorname{Var}(\mathrm{V})$ and $\operatorname{Var}(\mathrm{S})$ are functions of the particle-size distribution. This distribution typically ranges in terms of diameter over decades, from nanometers up to micrometers, and is not ideally captured by a small number of particles; therefore, it is obvious that the size distribution has an influence on the total uncertainty. In order to evaluate the variances, a discussion of the distributions of the number of particles $(\mathrm{N})$, the surface and the volume of particles (S and V) and the particle density $(\rho)$ is necessary. Since both surface and volume distribution of the particles are functions of the size distribution, we can discuss the more frequently used particle size distribution.

\section{Particle Size Distribution}

The size distribution of particles is frequently assumed to be lognormal (Hinds, 1999) in diameter, D, but can, in principle, have any shape. In order for Eqs. (2)-(3) to be valid, the variances of $\mathrm{S}$ and $\mathrm{V}$ must be finite, which is the case for any realistic distribution since diameter, surface and volume of particles are always finite. Since the size distribution of particles is not constant in the ambient air but depends on season, weather, nearby emissions and a 
variety of other environmental parameters (e.g., Väkevä et $a l ., 2000)$, it is not possible to assume one size distribution that is representative for all cases.

Given the size distribution, $p(D)$, as a function of the particles' diameter, D, we can write the expectation value and the variance of a variable being dependent on $\mathrm{D}$, such as the surface (S) and the volume (V), as in Eqs. (4)-(5), where $\mathrm{V}$ is used as an example.

$$
\begin{aligned}
& \langle V\rangle=\int_{0}^{\infty} p(D) V(D) d D \\
& \operatorname{Var}(V)=\int_{0}^{\infty} p(D)(V(D)-\langle V\rangle)^{2} d D
\end{aligned}
$$

Eqs. (4)-(5) show the expectation value, $\langle\mathrm{V}\rangle$, and the variance, $\operatorname{Var}(\mathrm{V})$, of a single particle's volume, which is only dependent on the distribution $\mathrm{p}(\mathrm{D})$. Since the variance of $\mathrm{S}$ and $\mathrm{V}$ is a function of this distribution, we can see that the distribution indeed affects the variability of the mass and the surface concentration of particulate matter directly. In order to evaluate Eqs. (4)-(5), a particle shape has to be assumed in order to find surface and volume as a function of diameter. The model is valid for any particle shape. Without any loss of generality, we choose spherical particle shapes for further evaluations for the sake of simplicity.

\section{Distribution of Particle Mass Density}

The distribution of the mass density, $\rho$, is - similar to the size distribution - dependent on various factors, such as the type of particle sources near the measurement. Measurement principles that do not directly determine mass - such as light scattering-need an assumption for the mean mass density to determine $\mathrm{PM}_{\mathrm{x}}$. An incorrect assumption of $\langle\rho\rangle$ directly affects the measured mass concentration, causing a systematic error. For the estimation of the total variability, we assume the mass density to be Gaussian distributed with a mean of $\langle\rho>$ and a variance of $\operatorname{Var}(\rho)$. If these cannot be experimentally evaluated, the parameters have to be estimated by using prior knowledge, such as knowledge of the nearby aerosol sources. Aerosol particles, in principle, may consist of any material, which implies a broad range of mass densities theoretically possible, ranging from a few hundred $\mathrm{kg} \mathrm{m}^{-3}$ up to over $19,000 \mathrm{~kg} \mathrm{~m}^{-3}$ for metallic particles. However, for realistic ambient cases, we can typically narrow down dominant particle densities. For example, Hand and Kreidenweis (2002) report a mass density of $1850 \pm 140 \mathrm{~kg} \mathrm{~m}^{-3}$ for $\mathrm{PM}_{2.5}$ measured in the southwest of Texas in the U.S.

\section{Particle Number}

For consistency, we require that $\left\langle\mathrm{PN}>=\mathrm{c}_{\mathrm{PN}},<\mathrm{PS}>=\mathrm{c}_{\mathrm{PS}}\right.$ and $\left\langle\mathrm{PM}_{\mathrm{x}}\right\rangle=\mathrm{c}_{\mathrm{PMx}}$, which means that the expectation value of each metric is equal to the respective ambient particle concentration on a large scale. We can use Eq. (2) to express the expectation value of the number of particles counted, $\langle\mathrm{N}>$, as stated in Eq. (6). While all three equations are valid, it makes sense to use only the equation with the corresponding ambient concentration of each metric for further evaluations.

$$
\begin{aligned}
& \langle N\rangle=c_{P N} V_{\text {Sample }} \\
& \langle N\rangle=\frac{c_{P S}}{\langle S\rangle} V_{\text {Sample }} \\
& \langle N\rangle=\frac{c_{P M x}}{\langle\rho\rangle\langle V\rangle} V_{\text {Sample }}
\end{aligned}
$$

We further assume uncorrelated particle positions, leading to uniformly distributed particles in space. Therefore, the total number of particles in a given volume will be Poisson distributed (Badger, 1946; Hinds, 1999). Although the assumption that particle positions are uncorrelated is under debate (Damit et al., 2014; Larsen, 2006), we draw the conclusion that the Poisson assumption is a valid starting point and neglect any possible correlations of particle positions, such as a clustering of particles of the same size or a complete avoidance of such clusters. In other words, we assume completely uniformly distributed particle positions. According to Larsen (2006), assuming that particles are randomly distributed in a volume, the Poisson distribution describes the number of particles within any subvolume.

Since we assume the number of particles within a sample is Poisson distributed, Eq. (7) expresses the variance of $\mathrm{N}$, as the variance of the Poisson distribution is equal to its expectation value.

$$
\operatorname{Var}(N)=\langle N\rangle
$$

\section{Coefficient of Variation}

In order to compare the uncertainties of the metrics, we use the coefficient of variation, which is the standard deviation divided by the expectation value and therefore shows the relative variability. The standard deviation of $\mathrm{PN}$, PS and $\mathrm{PM}_{\mathrm{x}}$ is given by the square root of the respective variance, as given in Eq. (3). This is the inherent variability of particle concentrations on an arbitrary scale and is therefore independent of the type of PM sensor, e.g., whether the sensor will analyze single particles and add them up, or take a cumulative measurement. The type of sensor used will, of course, influence the metric of the measurement and vice versa. The coefficient of variation of the measurement can be written as stated in Eq. (8) by using Eqs. (2), (3) and (7).

$$
\begin{aligned}
& \sigma_{P N, \text { rel }}=\frac{\sqrt{\operatorname{Var}(P N)}}{\langle P N\rangle}=\frac{1}{\sqrt{\langle N\rangle}} \\
& \sigma_{P S, \text { rel }}=\frac{\sqrt{\operatorname{Var}(P S)}}{\langle P S\rangle}=\frac{1}{\sqrt{\langle N\rangle}} \sqrt{1+\frac{\operatorname{Var}(S)}{\langle S\rangle^{2}}} \\
& \sigma_{P M x, \text { rel }}=\frac{\sqrt{\operatorname{Var}(P M x)}}{P M x}=\frac{1}{\sqrt{\langle N\rangle}} \sqrt{1+\frac{\operatorname{Var}(V)}{\langle V\rangle^{2}}+\frac{\operatorname{Var}(\rho)}{\langle\rho\rangle^{2}}}
\end{aligned}
$$


In Eq. (8), we can clearly see that the coefficient of variation of each metric considered drops as the sample size increases with a typical $1 / \sqrt{ } \mathrm{N}$ behaviour. As stated in Eq. (6), $<\mathrm{N}>$ is directly proportional to the sample volume, $\mathrm{V}_{\text {sample, }}$ and the mean ambient particle concentration in either metric. Therefore, the variability decreases for increasing sample volumes and higher concentrations, which both lead to better statistics. Another contribution to the variability of the measurement stems from the distribution of the particle surface for PS and the volume for $\mathrm{PM}_{\mathrm{x}}$, which are both directly related to the particle size distribution. In addition, the distribution of the mass density affects the variability of mass related metrics $\left(\mathrm{PM}_{\mathrm{x}}\right)$.

The formalism can easily be adapted to metrics such as $\mathrm{PM}_{10}, \mathrm{PM}_{2.5}$ or $\mathrm{PM}_{1}$. The upper bound of the metric influences the expectation value and variance of the volume of single particles $\langle\mathrm{V}>$ and $\operatorname{Var}(\mathrm{V})$. For example, the variance of particle sizes of $\mathrm{PM}_{1}$ is typically lower than for $\mathrm{PM}_{10}$ because the particles of interest expand over one more decade in diameter for $\mathrm{PM}_{10}$, as $1 \mu \mathrm{m}$ is the limit for $\mathrm{PM}_{1}$ and $10 \mu \mathrm{m}$ for $\mathrm{PM}_{10}$. Since the volume is proportional to the diameter cubed, the variance in volume is significantly affected by the particle sizes that are taken into account. Also, the majority of particles is typically below $1 \mu \mathrm{m}$ in diameter, which means that the Poisson counting uncertainty is not greatly affected by lowering the upper bound of the metric to $1 \mu \mathrm{m}$. For example, Klejnowski et al. (2013) report that during a measurement campaign in Zabrze in Poland, $99 \%$ of all particles by number concentration are below $1 \mu \mathrm{m}$ in diameter. Given a distribution, $\mathrm{p}(\mathrm{D})$, the variance for the metric of interest can be calculated by a normalization of $p(D)$ within the size window of interest. The probability outside this window is set to 0 . Then again, Eqs. (4) and (5) can be used to calculate expectation value and variance of a single particle's volume, respectively. Note that the ambient concentration, which is used to calculate the expectation value of the number of particles, $<\mathrm{N}>$, must therefore match the metric of interest.

\section{RESULTS AND DISCUSSION}

We begin our discussion with the uncertainty of particle number concentration measurements. This uncertainty is straightforward to calculate and to estimate for a measurement, as for a PN measurement, the number of particles counted has to be known obviously. Eq. (8) can be evaluated, which gives the uncertainty. The uncertainty of the measurement can be lowered by prolonging the measurement until a relative uncertainty is reached that is acceptable for the user.

For the particle surface concentration, the situation is already more complex, as can be seen in Eq. (8). There, the expectation value and the variance of a single particle's surface, which is a function of the particle size distribution, play a role. Depending on the measurement technique, the size distribution can be known or unknown. If there is no information on the particle size distribution, the exact uncertainty cannot be determined. Since the situation for mass concentrations, such as $\mathrm{PM}_{\mathrm{x}}$, is similar, and these metrics are the most common metrics for low-cost sensors, we focus the discussion on mass metrics.

As shown in Eq. (8) and Fig. 1, the coefficient of variation of the measured $\mathrm{PM}_{2.5}$ value is strongly dependent on the size distribution of particles. For this evaluation, we assume the particle diameter, $\mathrm{D}$, to be lognormally distributed and set $\rho$ to $1850 \pm 140 \mathrm{~kg} \mathrm{~m}^{-3}$ according to Hand and Kreidenweis (2002). For narrow particle size distributions, the variability is dominated by the Poisson contribution and the contribution from the mass density distribution. If we assume monodisperse particles, the geometric standard deviation (GSD) is 1 , and $\operatorname{Var}(\mathrm{V})$ is 0 . In this case, we are left with the Poisson uncertainty and the small contribution due to the mass density distribution. At broader particle size distributions with higher GSD, the size distribution related contribution dominates. Note that the standard deviations stemming from different sources do not add up, but the variances do, as shown in Eq. (3). The total coefficient of variation for the investigated case for $\mathrm{PM}_{2.5}$ ranges from roughly $4 \%$ to over $30 \%$ depending solely on the parameters of the size distribution. This major influence of the underlying particle size distribution makes the estimation of confidence intervals for a real measurement tricky. In a real case, users are forced to use the measured $\mathrm{PM}_{\mathrm{x}}$ value as an estimate for the ambient concentration. This is reasonable since the expectation value of the measurement equals the ambient concentration when there are no systematic errors in the measurement. If the measurement technique counts and sizes particles such as, for example, a light scattering device does, then Eq. (8) can be used to estimate the uncertainty. In this case, a mean mass density for the particles has to be assumed as well as a variance of the mass density. However, if the measurement technique does not provide information on the particle number and the size distribution, there is no estimate for the uncertainty of the measured $\mathrm{PM}_{\mathrm{x}}$ value as the coefficient of variation as stated in Eq. (8) cannot be evaluated. This is, for example, the case for gravimetric measurements. Also, the evaluation of Eq. (8) for a real measurement case will not lead to the exact uncertainty, as the information on the mean ambient concentration and the size distribution is not exact for finite sample sizes. In this work, we have all the information of the ambient situation and use it to find the variability of the particle concentrations. In a real case, there is little to no information on the mean ambient particle concentration, the size distribution and the mass density distribution of particles.

Mathematically speaking, the influence of the particle size distribution and the influence of the mass density distribution are equal. Nevertheless, we can state that for cases that are to be expected in the ambient air, the size distribution of particles dominates. This is reasonable since the particle size distribution ranges over more than three decades from nanometers to micrometers, while the mass density distribution can safely be assumed to extend over less than one decade. Therefore, the term $\operatorname{Var}(\rho) /<\rho>^{2}$ is, in general, small compared to $\operatorname{Var}(\mathrm{V}) /<\mathrm{V}>^{2}$.

Fig. 2 shows the uncertainty of $\mathrm{PM}_{1}, \mathrm{PM}_{2.5}$ and $\mathrm{PM}_{10}$ for lognormally distributed particles with an exemplary geometric mean (GM) of $0.3 \mu \mathrm{m}$ as a function of the 


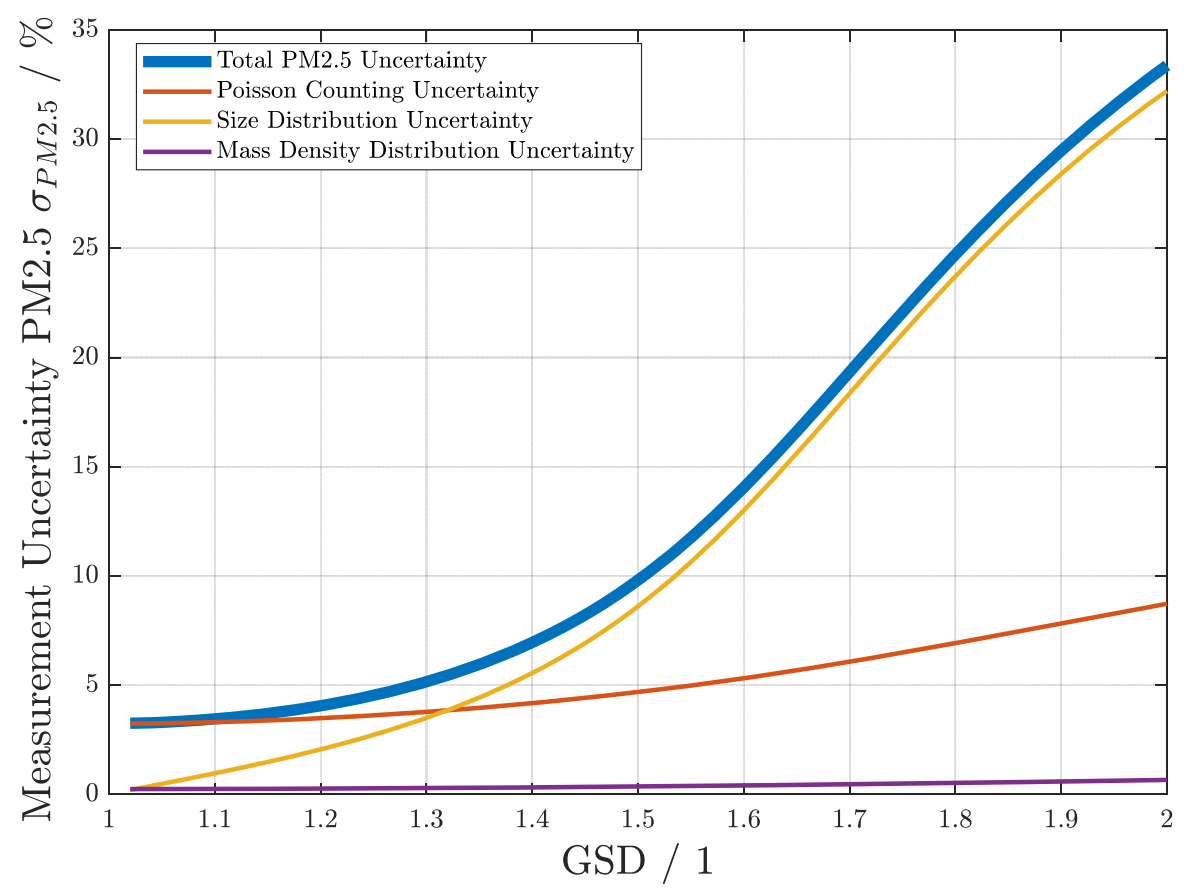

Fig. 1. Coefficient of variation as a function of the geometric standard deviation of the particle sizes for PM 2.5 . GSD corresponds to the width of the distribution. The calculation is done at an exemplary geometric mean of $300 \mathrm{~nm}$. For the calculation, only particles with diameters lower than $2.5 \mu \mathrm{m}$ are taken into account in order to show the uncertainty for $\mathrm{PM}_{2.5}$. The calculation is shown for a constant ambient $\mathrm{PM}_{2.5}$ concentration of $25 \mu \mathrm{g} \mathrm{m}^{-3}$, a particle mass density $<\rho>$ of $1850 \mathrm{~kg} \mathrm{~m}^{-3}$ with a variance $\operatorname{Var}(\rho)$ of $\left(140 \mathrm{~kg} \mathrm{~m}^{-3}\right)^{2}$ and a sample volume of $1 \mathrm{~mL}$. Although the ambient PM ${ }_{2.5}$ concentration is held constant, and therefore, the total mass that is sampled is also constant, the total uncertainty ranges over tens of percent. For realistic cases, the uncertainty stemming from the particle size distribution dominates. The Poisson contribution also rises as GSD rises since the number of particles at a constant ambient concentration gets lower as the variance of the size distribution rises.

geometric standard deviation (GSD) of the size distribution. The ambient mass concentration is set to $25 \mu \mathrm{g} \mathrm{m}^{-3}$ in each of the metrics. The relative uncertainty in general rises as GSD, and therefore the width of the particle size distribution, rises. At GSD values approximately lower than 1.5, the relative uncertainties of the metrics are equal, as the metrics do not differ from each other as long as all particles are below $1 \mu \mathrm{m}$ in diameter. For higher GSD values, the size distribution relevant to each of the metrics begins to differ, leading to a higher uncertainty of $\mathrm{PM}_{10}$ compared to $\mathrm{PM}_{2.5}$ and $\mathrm{PM}_{1}$. We can state that for the investigated parameters, the estimation of a mass concentration based on a small sample yields results that are more robust the lower the upper cutoff is. Since only a small number of particles typically has a diameter of greater than $2.5 \mu \mathrm{m}$ but their total volume is still of relevance, $\mathrm{PM}_{10}$ is especially prone to a high uncertainty.

Fig. 3 shows the measurement uncertainty of PN, PS and $\mathrm{PM}_{2.5}$ as a function of the sample volume. We assumed lognormally distributed particles with an exemplary GM of $0.3 \mu \mathrm{m}$ and a GSD of 1.7. The particle density, $\rho$, is set to $1850 \pm 140 \mathrm{~kg} \mathrm{~m}^{-3}$ according to Hand and Kreidenweis (2002). We only took particles with diameters below $2.5 \mu \mathrm{m}$ into account for this evaluation. In general, the relative uncertainty drops as the sample volume increases. The uncertainty of $\mathrm{PM}_{2.5}$ is the highest, followed by the uncertainty of the total particle surface (PS). The particle number concentration, PN, has the lowest uncertainty, which is immediately evident in Eq. (8), as for the uncertainty of $\mathrm{PM}_{\mathrm{x}}$ and PS, more terms are added. For lognormally distributed spherical particles, the relative uncertainty of the particle surface concentration is necessarily lower than the uncertainty of the volume concentration. The reason is that the surface is proportional to the square of the diameter of the particle, whereas the volume is proportional to the diameter cubed. This makes the mass metrics more prone to a relatively high uncertainty due to a small number of large particles. For the investigated parameters, a sample volume of $1 \mathrm{~mL}$ yields a relative uncertainty of $6 \%$ for $\mathrm{PN}, 11 \%$ for PS and $19 \%$ for $\mathrm{PM}_{2.5}$. Especially for $\mathrm{PM}_{2.5}$, this might be a high uncertainty, bearing in mind that it is only the inherent uncertainty of the measurement when the sensor's imperfections are yet to be considered. For a sample volume of $1 \mathrm{~L}$, the uncertainty below $1 \%$ for each metric is most likely overshadowed by the sensor's imperfections in a realistic case, which is why this uncertainty has never been observed using high-end laboratory equipment providing high flow rates and therefore has not been discussed in literature yet.

In Figs. 4 and 5, we show the relative uncertainty for $\mathrm{PM}_{2.5}$ as a function of the total mass that is sampled. As the uncertainty depends on the total number of particles that 


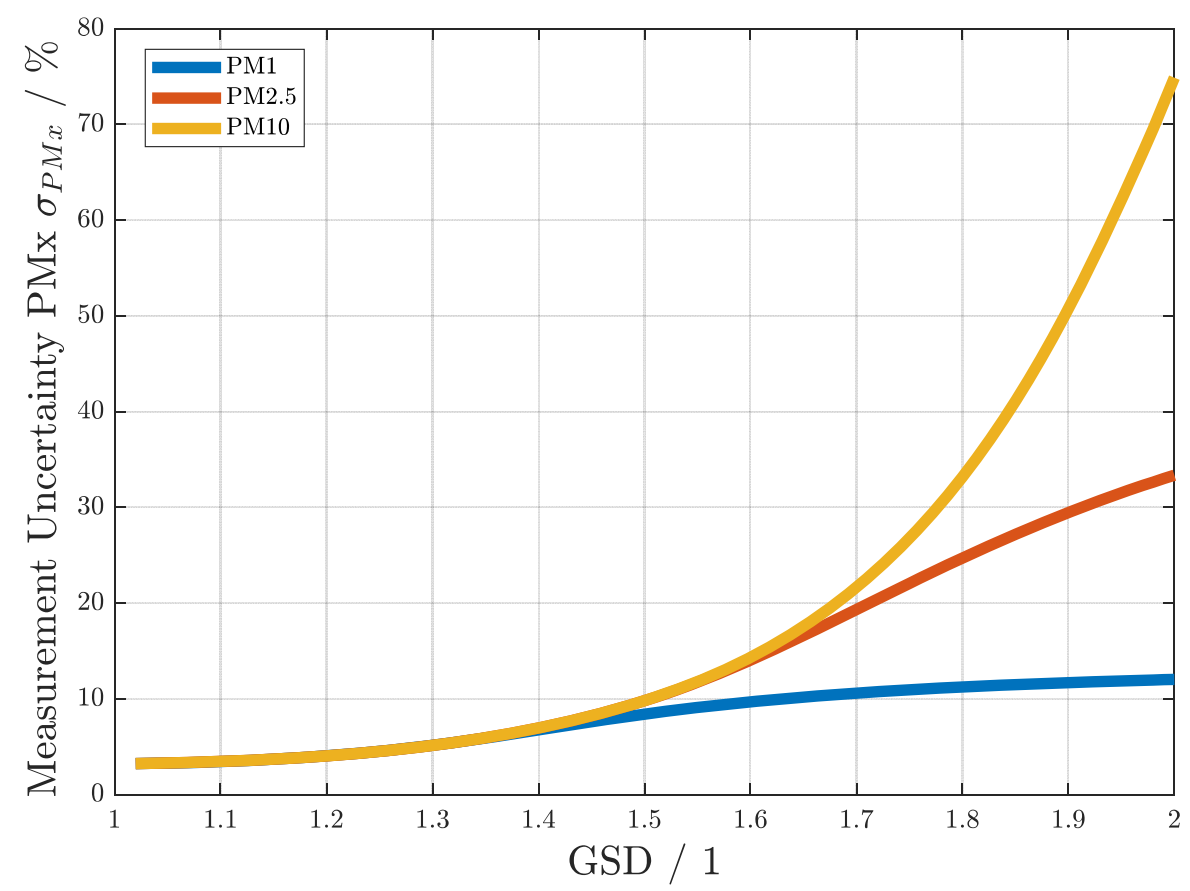

Fig. 2. Coefficient of variation as a function of the geometric standard deviation of the particle sizes for $\mathrm{PM}_{1}, \mathrm{PM}_{2.5}$ and $\mathrm{PM}_{10}$. GSD corresponds to the width of the distribution. The calculation is done at an exemplary geometric mean of $0.3 \mu \mathrm{m}$. The calculation is shown for an ambient particle mass concentration of $25 \mu \mathrm{g} \mathrm{m}^{-3}$ in both metrics, a particle mass density $<\rho>$ of $1850 \mathrm{~kg} \mathrm{~m}^{-3}$ with a variance $\operatorname{Var}(\rho)$ of $\left(140 \mathrm{~kg} \mathrm{~m}^{-3}\right)^{2}$ and a sample volume of $1 \mathrm{~mL}$. Although the ambient particle mass concentration is held constant in each metric, and therefore, the total mass that is sampled is also constant, the total uncertainty ranges over tens of percent and is a function of the upper cutoff of the relevant particle sizes. We can clearly see that at a GSD lower than $\sim 1.5$, the uncertainties are almost equal, as there are almost no particles that have a diameter above $1 \mu \mathrm{m}$, which makes the metrics' output equal. At higher GSDs, the metrics begin to differ, and so do the uncertainties. The lower the upper cutoff of the metric is, the lower the $\operatorname{Var}(\mathrm{V})$ of the relevant size window is. We can see that for equal distributions and similar mass concentrations, the relative uncertainties of $\mathrm{PM}_{1}, \mathrm{PM}_{2.5}$ and $\mathrm{PM}_{10}$ differ, although all of them take the mass into account.

are sampled, which is proportional to the sample volume times the ambient mass concentration, the total mass sampled by the sensor is a reasonable parameter. In Fig. 4, the uncertainty of $\mathrm{PM}_{2.5}$ is shown for lognormally distributed particles with a GM of $0.3 \mu \mathrm{m}$ and various GSD values. The particle density, $\rho$, is set to $1850 \pm 140 \mathrm{~kg} \mathrm{~m}^{-3}$ according to Hand and Kreidenweis (2002). In Fig. 5, the same is shown for a fixed GSD value of 1.5 and various values of GM. The relative uncertainty declines when higher total masses are sampled, which can be due to either a high sample volume, a high ambient particle mass concentration, or both. In general, the uncertainty rises for higher GSD values, as the size distribution becomes broader and therefore, the variance of the particles volume becomes higher. The uncertainty is also dependent on the geometric mean of the size distribution. The lower the GM, the more particles are necessary to have a fixed mass concentration.

Fig. 6 shows the relative uncertainty of $\mathrm{PM}_{1}, \mathrm{PM}_{2.5}$ and $\mathrm{PM}_{10}$ as a function of the total mass that is sampled at an exemplary lognormal particle size distribution with a GM of $0.3 \mu \mathrm{m}$ and a GSD of 1.7. The particle density, $\rho$, is set to $1850 \pm 140 \mathrm{~kg} \mathrm{~m}^{-3}$ according to Hand and Kreidenweis (2002). $\mathrm{PM}_{1}$ has the lowest cutoff and therefore the lowest relative uncertainty at equal masses that are sampled, whereas $\mathrm{PM}_{10}$ has the highest of the three.

As a rule of thumb, we propose that for $\mathrm{PM}_{2.5}$ and $\mathrm{PM}_{10}$, a minimum total mass of $100 \mathrm{pg}$ should be sampled, which yields a measurement uncertainty for $\mathrm{PM}_{2.5}$ of $\sim 10 \%$, as can be seen in Figs. 4 and 6. This assumes lognormally distributed particles with a geometric mean of $0.3 \mu \mathrm{m}$, a GSD of 1.7 and a particle density $(\rho)$ of $1850 \pm 140 \mathrm{~kg} \mathrm{~m}^{-3}$. We consider this a realistic worst-case scenario. At a $\mathrm{PM}_{2.5}$ mass concentration of $25 \mu \mathrm{g} \mathrm{m}^{-3}$, this would mean that approximately $4 \mathrm{~mL}$ of air have to be sampled; at $10 \mu \mathrm{g} \mathrm{m}^{-3}$, $10 \mathrm{~mL}$ would be necessary to stay below the $10 \%$ measurement uncertainty. For $\mathrm{PM}_{1}$, we propose a minimum of $30 \mathrm{pg}$ be sampled in order to have a relative uncertainty below $10 \%$. We would like to emphasize that this is not valid for arbitrary particle size distributions and mass density distributions. In order to evaluate the exact uncertainty, Eq. (8) has to be utilized.

For particle number measurements, the rule of thumb we propose is to count at least 100 particles, which yields a relative uncertainty of $10 \%$. Keeping in mind that imperfections of the measurement system itself come on top of this inherent uncertainty, a $10 \%$ inherent uncertainty may well be low compared to the uncertainties coming from technological boundaries. 


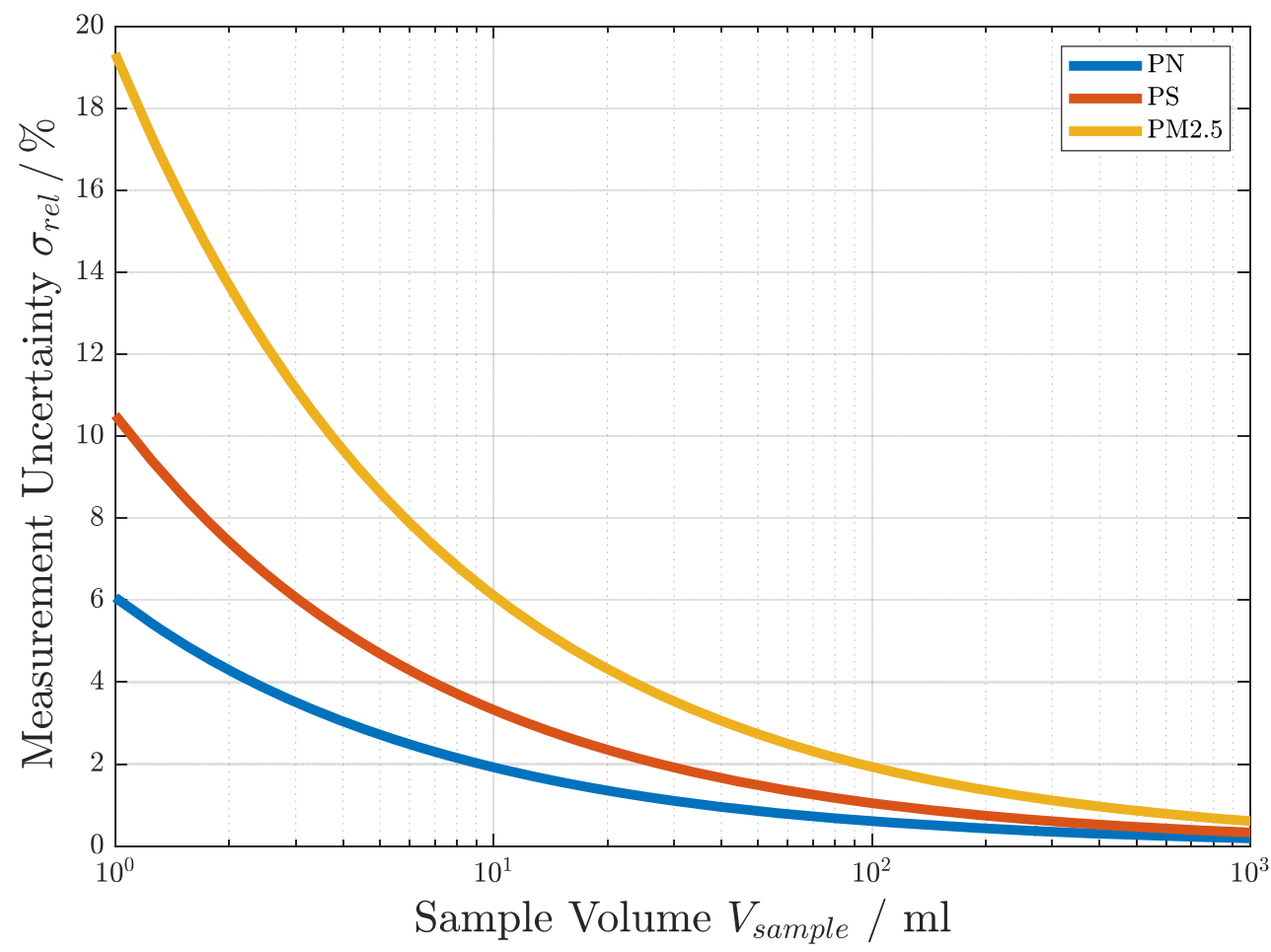

Fig. 3. The plot shows the measurement uncertainty as a function of the sample volume. The particle size distribution is assumed to be lognormal with an exemplary GM of $0.3 \mu \mathrm{m}$ and a GSD of 1.7. The ambient $\mathrm{PM}_{2.5}$ concentration is set to $25 \mu \mathrm{g} \mathrm{m}^{-3}$ and the particle density, $\rho$, to $1850 \pm 140 \mathrm{~kg} \mathrm{~m}^{-3}$ according to Hand and Kreidenweis (2002). $\sigma_{\text {rel }}$ drops as the sample volume increases for each of the metrics. Particles with diameters higher than $2.5 \mu \mathrm{m}$ are neglected for each metric. The relative uncertainty of $\mathrm{PN}$ is the lowest, followed by PS, and $\mathrm{PM}_{2.5}$ has the highest uncertainty.

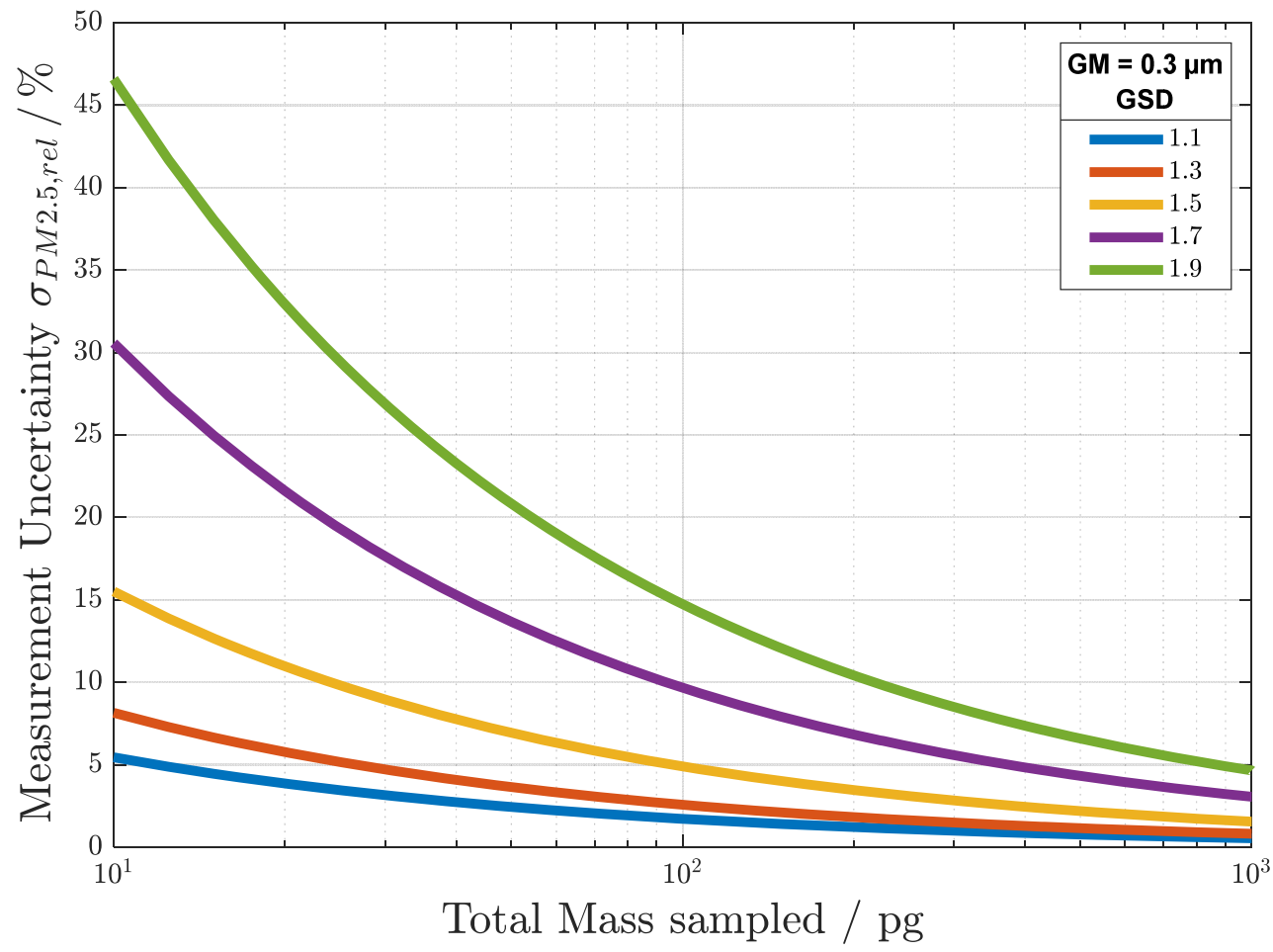

Fig. 4. The plot shows the relative uncertainty of $\mathrm{PM}_{2.5}$ as a function of the total mass that is sampled. A lognormal particle size distribution with an exemplary GM of $0.3 \mu \mathrm{m}$ is assumed. The results for various GSD values are shown. Assuming a $\mathrm{PM}_{2.5}$ concentration of $25 \mu \mathrm{g} \mathrm{m}{ }^{-3}$, a volume of $4 \mathrm{~mL}$ is typically necessary in order to sample a total mass of $100 \mathrm{pg}$. Depending on the GSD of the particle size distribution, the relative uncertainty of $\mathrm{PM}_{2.5}$ can differ significantly. 


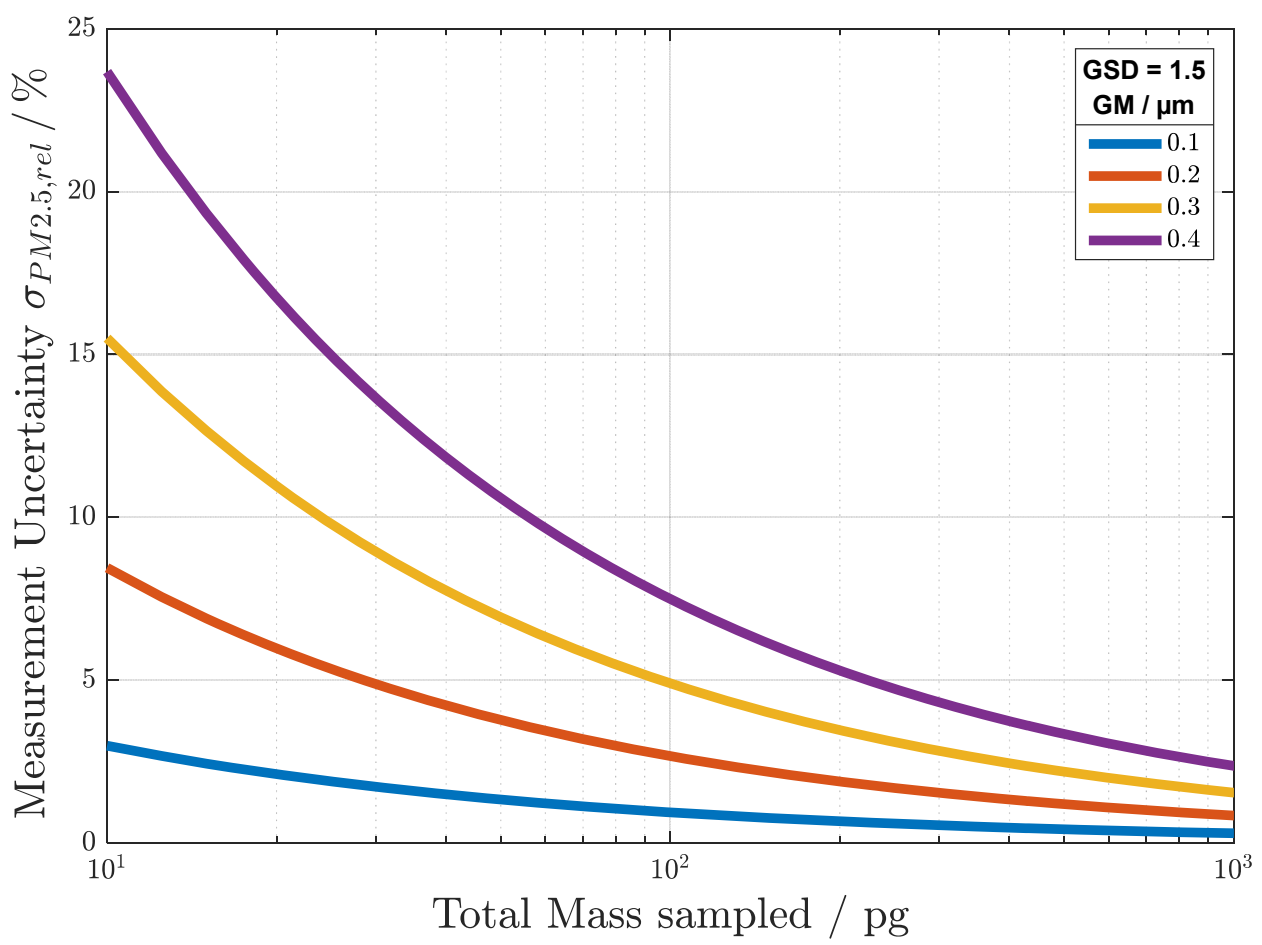

Fig. 5. The plot shows the relative uncertainty of $\mathrm{PM}_{2.5}$ as a function of the total mass that is sampled. A lognormal particle size distribution with a GSD of 1.5 is assumed. The results for various GM values are shown. Depending on the $\mathrm{GM}$, the relative uncertainty of $\mathrm{PM}_{2.5}$ can differ significantly. This is reasonable since at lower GM values, a greater number of particles is necessary to have the same mass concentration as for higher GM values. The greater number of particles lowers the relative uncertainty.

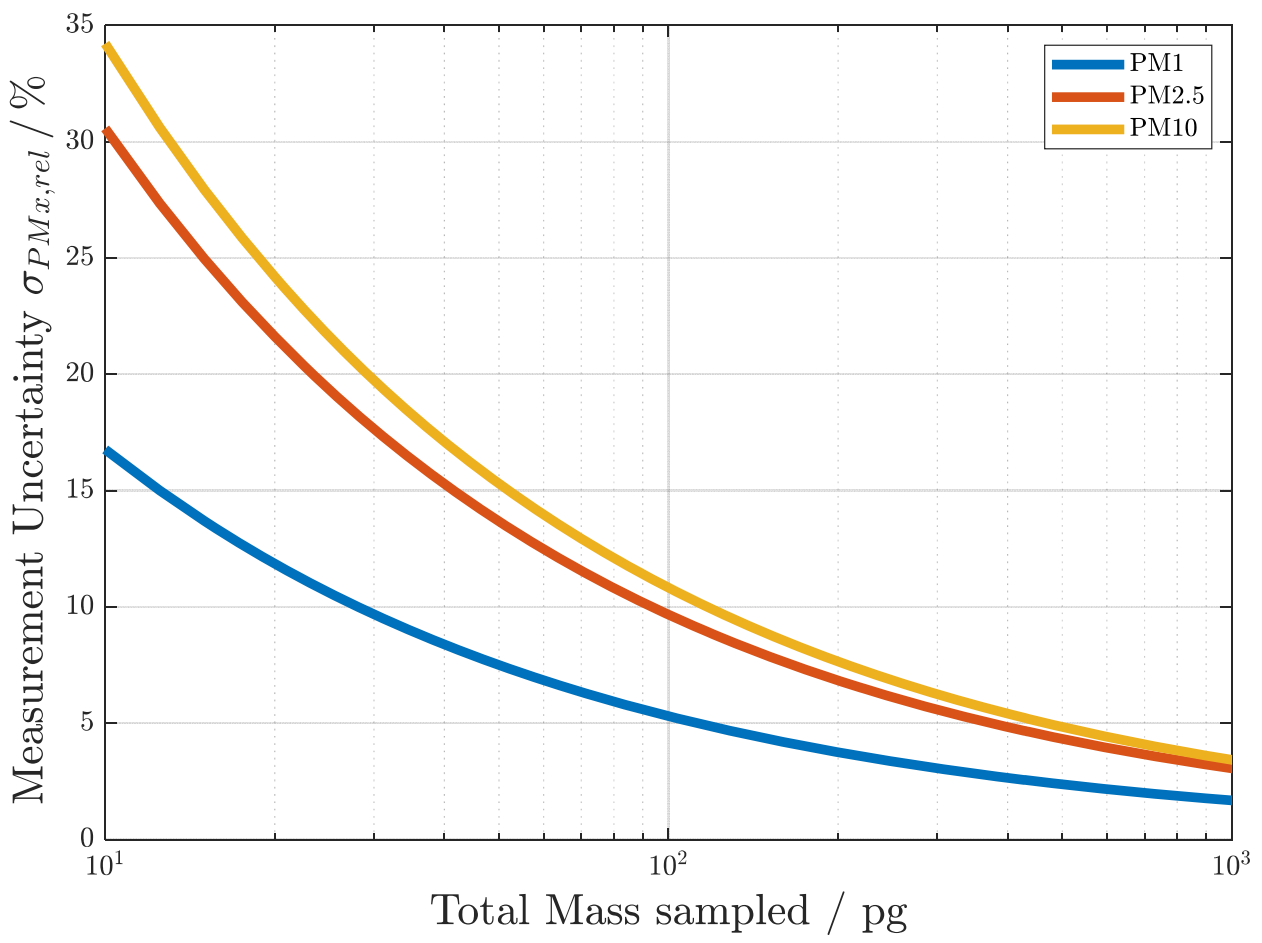

Fig. 6. The plot shows the relative uncertainty of $\mathrm{PM}_{1}, \mathrm{PM}_{2.5}$ and $\mathrm{PM}_{10}$ as a function of the total mass that is sampled. For each of the metrics, the same lognormal particle size distribution with a GM of $0.3 \mu \mathrm{m}$ and a GSD of 1.7 is assumed. The relative uncertainties of $\mathrm{PM}_{1}, \mathrm{PM}_{2.5}$ and $\mathrm{PM}_{10}$ differ due to the upper cutoff in particle size that is considered. With a total sampled mass of $100 \mathrm{pg}$, the uncertainty is $5 \%$ for $\mathrm{PM}_{1}, 10 \%$ for $\mathrm{PM}_{2.5}$ and $11 \%$ for $\mathrm{PM}_{10}$. For $\mathrm{PM}_{1}, 30$ pg are sufficient for the assumed parameters to have a relative uncertainty below $10 \%$. 


\section{CONCLUSIONS}

We have established a model for estimating the local variability of particulate matter concentrations on arbitrarily small scales and evaluated it using various parameters. The model addresses the uncertainty in the particle number concentration and the surface concentration as well as the mass concentration for metrics such as $\mathrm{PM}_{1}, \mathrm{PM}_{2.5}$ and $\mathrm{PM}_{10}$. The variability is due to the small number of particles within a small sample, which is used to represent a rather complex distribution of particle sizes and mass densities. Although we assume that variability on a small scale has no influence on human health, it does have a major impact on the validity of miniaturized PM sensor measurements. We find that even a sensor without any imperfections is barely able to assign a reliable particle concentration to the ambient environment when the sample volume is on the order of milliliters. Depending on the metric used, the uncertainty depends on the distributions of the influencing parameters. The overall uncertainty for the particle number concentration depends on the total particle concentration and the sample volume; additionally, the particle surface concentration is dependent on the size distribution, and for mass-related metrics such as $\mathrm{PM}_{1}$, $\mathrm{PM}_{2.5}$ and $\mathrm{PM}_{10}$, also the mass density distribution has to be taken into account. These values, aside from the sample volume, may remain unknown with low-cost sensors and low sample volumes, effectively preventing the estimation of the uncertainty in the measurement. The total uncertainty is on the order of $10 \%$ for realistic cases and a sample volume of a few milliliters.

We have shown that for the same ambient conditions, the relative uncertainty in the particle number is lower than that in the surface concentration, which is lower than the relative uncertainty in the mass concentration. A small sample volume is therefore better suited to estimating number or surface concentrations than ambient mass concentrations. When mass metrics are used, the uncertainty is typically lower for estimating $\mathrm{PM}_{1}$ than $\mathrm{PM}_{2.5}$. The highest relative uncertainty is generally associated with $\mathrm{PM}_{10}$, as the range of measured particle sizes expands to include the largest diameters.

Miniaturized sensors with small footprints can overcome these limitations only by allowing relatively long measurement times or providing high flow rates. These measures, however, are incompatible with miniaturization since both features increase energy consumption and a high flow rate usually requires a large footprint. On top of these inherent limitations, sensor imperfections must be taken into account. As a rule of thumb for $\mathrm{PM}_{2.5}$ and $\mathrm{PM}_{10}$, we propose a minimum total sample mass of $100 \mathrm{pg}$ - typically $4 \mathrm{~mL}$ of sampled air at an ambient mass concentration of $25 \mu \mathrm{g} \mathrm{m}^{-3}$ - in order to cap the relative uncertainty at approximately $10 \%$ for cases we consider to be realistic for ambient measurements; to obtain a $\sim 10 \%$ uncertainty for $\mathrm{PM}_{1}$, a minimum total sample mass of $30 \mathrm{pg}$ is required. The miniaturization of particle sensors may hit a boundary once sample volumes reach $\sim 1 \mathrm{~mL}$ due to the inherent statistical variability of particle concentrations on such small volumes. In the opinion of the authors, a sample volume of less than $1 \mathrm{~mL}$ at realistic ambient particle concentrations may be insufficient for providing any reliable results. This handicap is especially severe when measuring mass-related metrics, such as $\mathrm{PM}_{1}, \mathrm{PM}_{2.5}$ and $\mathrm{PM}_{10}$.

\section{ACKNOWLEDGMENTS}

This work was funded by FFG (Austrian Research Promotion Agency) Grant \#86197 and ams AG.

Supported by TU Graz Open Access Publishing Fund.

\section{SUPPLEMENTARY MATERIAL}

Supplementary data associated with this article can be found in the online version at http://www.aaqr.org.

\section{REFERENCES}

Badger, E.H.M. (1946). Particle counts in the ultramicroscope. Nature 157: 480.

Bao, Y., Cai, S., Yu, H., Xu, T., Xu, P. and Li, X. (2018). A resonant cantilever based particle sensor with particle-size selection functiqn. J. Micromech. Microeng. 28: 085019.

Ciccarella, P., Carminati, M., Sampietro, M. and Ferrari, G. (2016). Multichannel 65zF RMS resolution CMOS monolithic capacitive sensor for counting single micrometer-sized airborne particles on chip. IEEE $J$. Solid-State Circuits 51: 2545-2553.

Damit, B., Wu, C. and Cheng, M. (2014). On the validity of the poisson assumption in sampling nanometer-sized aerosols. Aerosol Sci. Technol. 48: 562-570.

Hand, J.L. and Kreidenweis, S.M. (2002). A new method for retrieving particle refractive index and effective density from aerosol size distribution data. Aerosol Sci. Technol. 36: 1012-1026.

Hapidin, D. A., Saputra, C., Maulana, D. S., Munir, M. M., and Khairurrijal, K. (2019). Aerosol chamber characterization for commercial particulate matter (PM) sensor evaluation. Aerosol Air Qual. Res. 19: 181-194.

Hinds, W.C. (1999). Aerosol technology: Properties, behavior, and measurement of airborne particles. Wiley, New York, USA.

Kioumourtzoglou, M., Schwartz, J.D., Weisskopf, M.G., Melly, S J., Wang, Y., Dominici, F. and Zanobetti, A. (2016). Long-term $\mathrm{PM}_{2.5}$ exposure and neurological hospital admissions in the northeastern United States. Environ. Health Perspect. 124: 23-29.

Klenke, A. (2013). Wahrscheinlichkeitstheorie. 3. Auflage. Springer Spektrum, Berlin-Heidelberg, Germany, pp. 103-109

Larsen, M.L. (2007). Spatial distributions of aerosol particles: Investigation of the Poisson assumption. $J$. Aerosol Sci. 38: 807-822.

Li, X., Iervolino, E., Santagata, F., Wei, J., Yuan, C.A., Sarro, P.M. and Zhang, G.Q. (2014). Miniaturized particulate matter sensor for portable air quality monitoring devices. IEEE Sensors 2014 - Valencia 
Conference Centre, Valencia, Spain, pp. 2151-2154.

Linden, W., Dose, V. and Toussaint, U. (2014). Bayesian probability theory: Applications in the physical sciences. Cambridge University Press, Cambridge, UK.

Paprotny, I., Doering, F., Solomon, P.A., White, R.M. and Gundel, L.A. (2013). Microfabricated air-microfluidic sensor for personal monitoring of airborne particulate matter: Design, fabrication, and experimental results. Sens. Actuators, A 201: 506-516.

Ranft, U., Schikowski, T., Sugiri, D., Krutmann, J. and Krämer, U. (2009). Long-term exposure to traffic- related particulate matter impairs cognitive function in the elderly. Environ. Res. 109: 1004-1011.

Väkevä, M., Hämeri, K., Puhakka, T., Nilsson, E.D., Hohti, H. and Mäkelä, J.M. (2000). Effects of meteorological processes on aerosol particle size distribution in an urban background area. J. Geophys. Res. 105: 9807-9821.

Received for review, January 30, 2019

Revised, May 9, 2019

Accepted, May 11, 2019 